The bracket in Eq. (4) is positive, if the speed increases as one moves from $P$ in the direction of the resulting velocity to a neighboring point $P_{1}$ on the stream line through $P$. If the images of the points $P$ and $P_{1}$ in the hodograph plane are denoted by $P^{\prime \prime}$ and $P_{1}^{\prime \prime}$, the bracket in (4) is therefore positive, if $P^{\prime \prime}$ is closer to the origin $O^{\prime \prime}$ of the hodograph plane than $P_{1}^{\prime \prime}$. As the signs of $u$ and $v$ are readily determined, we have therefore a convenient criterion for the sign of the right-hand side of (4), that is for the sign of the plastic power. For a steady flow, in particular, this criterion requires that the particle that is instantaneously at $P$ should be accelerating or decelerating according to whether $u$ and $v$ have the same signs or not.

\title{
BibLiOGRAPHY
}

1. W. Prager, Trans. Roy. Inst. Technology, Stockholm, No. 65 (1953)

2. A. P. Green, J. Mech. Phys. Solids 2, 73, 296 (1954)

\section{NECESSARY AND SUFFICIENT CONDITIONS FOR THE EXISTENCE OF NEGATIVE SPECTRA*}

\author{
By C. R. PUTNAM (Purdue University)
}

Let $f(t)$ be a real-valued continuous function on the $t$-interval $0 \leqq t \leqq 2 T$ and let $x=x(t)$ be a real-valued solution $(\not \equiv 0)$ of the differential equation

$$
x^{\prime \prime}+f(t) x=0 \text {. }
$$

If $f^{+}=f$ or 0 according as $f \geqq 0$ or $f<0$, and if $x(a)=x(b)=0$, where $a<b$, then there holds the inequality

$$
\int_{a}^{b} f^{+}(t) d t>4 /(b-a)
$$

due essentially to Liapounoff (see [1]; also [8]). Moreover, according to [4] (see also [1]), the constant 4 of (2) is the best possible, in the sense that (2) need not hold (for arbitrary $f$ ) if the 4 is replaced by $4+\epsilon$, where $\epsilon>0$. Hence, it is easy to see that the inequality

$$
T \int_{0}^{T}\left[f^{+}(t)+f^{+}(2 T-t)\right] d t \quad\left(=T \int_{0}^{2 T} f^{+}(t) d t\right)>2
$$

is necessary, but $T \int_{0}^{2 T} f^{+}(t) d t>2+\epsilon$ is not, in order that the Sturm-Liouville boundary value problem

$$
L(x)+\lambda x=0 \quad\left[L(x) \equiv x^{\prime \prime}+f x\right], \quad x(0)=x(2 T)=0
$$

possess an eigenvalue $\lambda<0$ (or even $\leqq 0$ ).

It will be shown in this note that there is a sufficient criterion similar to (3). In fact,

*Received June 11, 1954. This work was supported by the National Science Foundation research grant NSF-G-481. 
the following will be proved: If $f(t)$ satisfies the inequality

$$
M(f) \equiv T^{-1} \int_{0}^{T} t^{2}[f(t)+f(2 T-t)] d t>2,
$$

then the spectrum of the boundary value problem (4) possesses some eigenvalue $\lambda<0$. Furthermore, the constant 2 occurring on the right sic'c of (5) is the best possible in the sense that for every $\epsilon>0$, there is a continuous function $f(t)$ satisfying $M(f)>2-\epsilon$, and such that the values $\lambda$ in the spectrum of the eigenvalue problem (4) satisfy $\lambda>0$. ( Note that the similarity between (3) and (5) is particularly apparent if $f(t) \geqq 0$, so that $f=f^{+}$.)

If one grants, for the present, the italicized assertion, the proof of the claim following it is easy. In fact, let a continuous function $f(t)$ be defined on $0 \leqq t \leqq 2 T$ so that $f(t) \geqq 0$ and so that

$$
2-\epsilon<M(f)<2-\epsilon / 2 \quad \text { and } \quad\left|M(f)-T \int_{0}^{2 T} f(t) d t\right|<\epsilon / 2 .
$$

Clearly this can be done; one need only define $f$ to be 0 everywhere on $0 \leqq t \leqq 2 T$ except on some sufficiently small interval about $T$. Then the spectrum of the boundary value problem (5), for the above constructed $f$, consists of positive eigenvalues only. In fact, if there were an eigenvalue $\lambda \leqq 0$, there would exist a solution $x(t) \not \equiv 0$ of (1) satisfying $x(0)=x\left(T_{1}\right)=0,0<T_{1} \leqq 2 T$. Consequently, relation (2) for $a=0$ and $b=T_{1}$ would now imply (3), that is $T \int_{0}^{2 T} f(t) d t>2$, in contradiction with (6).

There remains then to prove the italicized assertion. To this end, suppose, if possible, that there does not exist a negative eigenvalue, so that, in view of the Parseval equality, the relation

$$
\int_{0}^{2 T}\left(x^{\prime 2}-f x^{2}\right) d t=-\int_{0}^{2 T} x L(x) d t=\sum \lambda_{k} c_{k}^{2} \geqq 0
$$

holds for all real-valued functions $x$ possessing continuous second derivatives on $0 \leqq t \leqq 2 T$ and satisfying $x(0)=x(2 T)=0$. Here, the constants $c_{k}$ are defined by $c_{k}=\int_{0}^{2} x \phi_{k} d t$, where $\phi_{k}$ is the normalized eigenfunction belonging to the eigenvalue $\lambda_{k}$. (In connection with (7), see [3], p. 392 and the reference given there to Hamel [2]. For applications of an inequality similar to (7) but relating to singular boundary value problems [7] on the half-line $0 \leqq t<\infty$, see, e.g., [5], [6].) Next, define the continuous function $y(t)$ on $0 \leqq t \leqq 2 T$ by putting $y(t)=t$ on $0 \leqq t \leqq T$ and $y(t)=2 T-t$ on $T \leqq t \leqq 2 T$. (See [1], p. 69.) Then

$$
\int_{0}^{2 T}\left(y^{\prime 2}-f y^{2}\right) d t=2 T-\int_{0}^{T} t^{2} f(t) d t-\int_{T}^{2 T}(2 T-t)^{2} f(t) d t .
$$

It follows from (5) that

$$
\int_{0}^{2 T}\left(y^{\prime 2}-f y^{2}\right) d t<0 .
$$

Clearly, the function $y$ can be approximated by functions $x$ which possess continuous second derivatives on $0 \leqq t \leqq 2 T$, satisfy $x(0)=x(2 T)=0$, and which violate the inequality of (7). This violation yields a contradiction and shows that the assumption that the spectrum contains no negative eigenvalues is untenable. The proof of the italicized assertion is thus complete. 


\section{REFERENCES}

[1] G. Borg, On a Liapounoff criterion of stability, Am. J. Math. 71, 67-70 (1949)

[2] G. Hamel, Ueber das infinitäre Verhalten der Integrale einer linearen Differentialgleichung zweiter Ordnung, wenn die characterische Gleichung zwei gleiche Wurzeln hat, Math. Z., 1, 220-228 (1918)

[3] P. Hartman, On non-oscillatory differential equations of second order, Am. J. Math. 74, 389-400 (1952)

[4] E. R. van Kampen and A. Wintner, On an absolute constant in the theory of variational stability, Am. J. Math. 59, 270-274 (1937)

[5] C. R. Putnam, An oscillation criterion involving a minimum principle, Duke Math. J. 16, 633-636 (1949)

[6] C. R. Putnam, On the least eigenvalue of Hill's equation, Q. Appl. Math. 9, 310-314 (1951)

[7] H. Weyl, Ueber gewöhnliche Differentialgleichungen mit Singularitäten und die zugehörigen Entwicklungen willkürlicher Funktionen, Math. Ann. 68, 220-269 (1910)

[8] A. Wintner, On the non-existence of conjugate points, Am. J. Math. 73, 368-380 (1951)

\section{Correction to my paper}

\section{A NEW SINGULARITY OF TRANSONIC PLANE FLOWS*}

Quarterly of Applied Mathematics, XII, 343-349 (1955)

By A. R. MANWELL (University College, Swansea)

A much more detailed study of the singular solution discussed rather briefly in the note of the above title has shown that several statements in Sec. 4 are incorrect. Briefly, the expansions (4.4) and so also (4.5), (4.6) are valid only locally for either $\theta=0$ or for $\theta=\pi$, but not necessarily for both. We may not infer from these expansions the existence of solutions in the whole interval $(0, \pi)$. (In particular, on account of the pole at $Z=1$, we may not replace in (4.3) a contour for which $Z-1=2 i \exp (i \theta) \sin \theta$ is very small by the unit circle $Z=1$ ).

A correct discussion shows that (4.3) and (4.4) yield only two independent solutions. As a consequence, the singular solution can be smoothly continued across the sonic line for $\theta>0$ but, unless we admit further singularities in the supersonic region, the flow would not join up smoothly for $\theta<0$. Since we are seeking possible criteria for the breakdown of flow solutions, this correction leads to a slight strengthening of our original conclusion.

*Received March 7, 1955.

\section{A MINIMUM PRINCIPLE OF PLASTICITY*}

\section{BY D. TRIFAN (University of Arizona)}

This note is concerned with the removal of a certain restriction imposed by a proof ${ }^{1}$ [Sec. 5] of a minimum principle of an isotropic, incompressible, strain-hardening material exhibiting a gradual transition from the elastic to the plastic state. The governing stressstrain relation for loading is given by

$$
s_{i j}^{*}=2 G_{0} \epsilon_{i j}^{*}-p(E) \epsilon_{i j} E^{*},
$$

*Received Feb. 28, 1955.

'Section numbers enclosed in brackets refer to the following paper: D. Trifan, $A$ new theory of plastic flow, Q. Appl. Math. 7, pp. 201-211 (1949). 